\title{
New Blockcipher Modes of Operation with Beyond the Birthday Bound Security
}

\author{
Tetsu Iwata \\ Dept. of Computer and Information Sciences, \\ Ibaraki University \\ 4-12-1 Nakanarusawa, Hitachi, Ibaraki 316-8511, Japan \\ iwata@cis.ibaraki.ac.jp \\ http://crypt.cis.ibaraki.ac.jp/
}

\begin{abstract}
In this paper, we define and analyze a new blockcipher mode of operation for encryption, CENC, which stands for Cipher-based ENCryption. CENC has the following advantages: (1) beyond the birthday bound security, (2) security proofs with the standard PRP assumption, (3) highly efficient, (4) single blockcipher key, (5) fully parallelizable, (6) allows precomputation of keystream, and (7) allows random access. CENC is based on the new construction of "from PRPs to PRF conversion," which is of independent interest. Based on CENC and a universal hash-based MAC (Wegman-Carter MAC), we also define a new authenticated-encryption with associated-data scheme, CHM, which stands for CENC with Hash-based MAC. The security of CHM is also beyond the birthday bound.
\end{abstract}

\section{Introduction}

A blockcipher mode of operation, or a mode for short, is an algorithm that provides security goals, such as privacy and/or authenticity, based on blockciphers. The mode for privacy is called an encryption mode.

Of many encryption modes, counter (CTR) mode has a number of desirable advantages, and it works as follows. Let $E$ be a blockcipher whose block length is $n$ bits, and let ctr be an $n$-bit counter. For a plaintext $M=\left(M_{0}, \ldots, M_{l-1}\right)$ broken into $n$-bit blocks, let

$$
\left\{\begin{array}{l}
C_{i} \leftarrow M_{i} \oplus S_{i}, \text { where } S_{i} \leftarrow E_{K}(\operatorname{ctr}+i) \text { for } 0 \leq i \leq l-1, \\
\operatorname{ctr} \leftarrow \operatorname{ctr}+l .
\end{array}\right.
$$

The ciphertext is $C=\left(C_{0}, \ldots, C_{l-1}\right)$, and $S=\left(S_{0}, \ldots, S_{l-1}\right)$ is the keystream.

Starting from [3], provable security (or reduction-based security) is the standard security goal for modes. For encryption modes, we consider the strong security notion of privacy called "indistinguishability from random strings" from 23, which provably implies the more standard notions given in 11. In this strong notion, the adversary is in the adaptive chosen plaintext attack scenario, and the goal is to distinguish the ciphertext from the random string of the same length (where ctr is not considered part of the ciphertext). 
For CTR mode, Bellare, Desai, Jokipii and Rogaway were the first who presented the proof of security [1. The nonce-based treatment of CTR mode was presented by Rogaway 21. It was proved that, for any adversary against CTR mode, the success probability is at most $0.5 \sigma(\sigma-1) / 2^{n}$ under the assumption that the blockcipher is a secure pseudorandom permutation (PRP), where $\sigma$ denotes the total ciphertext length in blocks that the adversary obtains. This is the well-known birthday bound.

The above analysis is tight. There is an adversary that meets the security bound within a constant factor. The adversary simply searches for a collision in the keystream of $\sigma$ blocks, and guesses the data is the true ciphertext iff there is no collision. It is easy to show that the success probability is at least $0.3 \sigma(\sigma-1) / 2^{n}$. This implies that, as long as $E_{K}(\cdot)$ is a permutation, there is no hope that CTR mode achieves beyond the birthday bound security.

In this paper, we design a new blockcipher mode of operation for encryption. The goals are: (1) beyond the birthday bound security, (2) security proofs with the standard PRP assumption, (3) highly efficient, (4) single blockcipher key, (5) fully parallelizable, (6) allows precomputation of keystream, and (7) allows random access. The original CTR mode achieves all the above goals except for the first one, while we improve the security of CTR mode without breaking its important advantages. As for the security assumption, we do not use the ideal blockcipher model. For efficiency, the number of blockcipher calls is close to CTR mode, and we avoid using any heavy operations, e.g., re-keying.

Now in CTR mode, it is known that if $E_{K}(\cdot)$ is a secure pseudorandom function $(\mathrm{PRF})$, then for any adversary the success probability 0 , well beyond the birthday bound. Thus the natural approach to achieve beyond the birthday bound security is to construct a secure PRF from PRPs and use the PRF in CTR mode, where the security of PRF must be beyond the birthday bound. There are several such constructions 410162. The first construction, due to Bellare, Krovetz, and Rogaway is called data-dependent re-keying [4]. It was proved that the construction achieves beyond the birthday bound security in the ideal blockcipher model. The truncation construction was analyzed by Hall et. al., and they also considered the order construction [10. Lucks [16] and Bellare and Impagriazzo [2] independently analyzed the construction $G_{K}(x)=E_{K}(x \| 0) \oplus E_{K}(x \| 1)$, where $x \in\{0,1\}^{n-1}$. Lucks also considered a more generalized construction where $d$ blockciphers are xor'ed to output an $n$-bit block, and a multiple key version, $G_{K_{1}, K_{2}}(x)=E_{K_{1}}(x) \oplus E_{K_{2}}(x)$, where $x \in\{0,1\}^{n}$ [16].

By using these constructions in CTR mode, it is possible to construct encryption modes with beyond the birthday bound. However, there is a significant restriction in efficiency, and/or it breaks several important advantages of the original CTR mode. For example, if the construction from 4] is used, we need the ideal blockcipher model for security proofs and have the efficiency problem for re-keying. The constructions from [10] are not very efficient and the truncation construction has relatively small security improvement. If $G_{K}(x)=$ $E_{K}(x \| 0) \oplus E_{K}(x \| 1)$ is used, $2 l$ blockcipher calls are needed to encrypt $l$ plaintext 
blocks. We see that the main reason for inefficiency is that the output size of these PRFs is one block (or less).

To achieve beyond the birthday bound security, we first show a new "from PRPs to PRF conversion," where the output size of the new PRF is larger than the block size. In particular, our PRF outputs $w$ blocks at a time by using $w+1$ blockcipher calls. The parameter, $w$, is called a frame width, and one frame is equivalent to $n w$ bits. The frame width, $w$, can be any fixed positive integer. We prove that the adversary's success probability is at most $w \sigma^{3} / 2^{2 n-3}+w \sigma / 2^{n}$, where $\sigma$ is the total number of blocks that the adversary obtains.

Based on the PRF, we show a new encryption mode with beyond the birthday bound security. The new mode is called CENC, which stands for Cipher-based ENCryption. CENC calls $l+\lceil l / w\rceil$ blockciphers to encrypt $l$ plaintext blocks, and the default value is $w=2^{8}$, i.e., we need $l+\lceil l / 256\rceil$ blockcipher calls to encrypt $l$ plaintext blocks. Notice that, with the AES $(n=128)$, one frame corresponds to $n w$ bits, which is $128 \times 256=4 \mathrm{KBytes}$, and almost all the traffic on the Internet fits in one frame [8]. This implies we need $l+1$ blockcipher calls for these short data, i.e., the cost is one blockcipher call per data compared to CTR mode. As for the security, with $w=2^{8}$ and the AES, the security bound of CENC is $\hat{\sigma}^{3} / 2^{248}+\hat{\sigma} / 2^{121}$, where $\hat{\sigma}$ is (roughly) the total number of blocks that the adversary obtains. The security of CENC is beyond the birthday bound with the standard PRP assumption. Besides, CENC has desirable advantages of CTR mode. It uses single blockcipher key, it is fully parallelizable, allows precomputation of keystream, and random access is possible.

An authenticated-encryption with associated-data scheme, or AEAD scheme, is a scheme for both privacy and authenticity. It takes a plaintext $M$ and a header $H$, and provides privacy for $M$ and authenticity for both $M$ and $H$. There are a number of proposals: we have IAPM [13], OCB mode [23], CCM mode [2512], EAX mode [7, CWC mode [15], GCM mode [1920], and CCFB mode [17]. Based on CENC and a universal hash-based MAC (Wegman-Carter MAC), we propose a new AEAD scheme called CHM, which stands for CENC with Hash-based MAC. We show that the security of CHM is beyond the birthday bound, which is the first example in literature. The scheme is similar to GCM, achieves higher security with small efficiency loss. It also fixes several undesirable properties of GCM (for example, GCM is not online in the sense that headers must be MACed before starting MAC the ciphertext, and the plaintext length is limited to 64GBytes when used with the AES).

\section{Preliminaries}

Notation. If $x$ is a string then $|x|$ denotes its length in bits. If $x$ and $y$ are two equal-length strings, then $x \oplus y$ denotes the xor of $x$ and $y$. If $x$ and $y$ are strings, then $x \| y$ denotes their concatenation. Let $x \leftarrow y$ denote the assignment of $y$ to

$x$. If $X$ is a set, let $x \stackrel{R}{\leftarrow} X$ denote the process of uniformly selecting at random an element from $X$ and assigning it to $x$. For a positive integer $n,\{0,1\}^{n}$ is the set of all strings of $n$ bits. For positive integers $n$ and $w,\left(\{0,1\}^{n}\right)^{w}$ is the set of 
all strings of $n w$ bits, and $\{0,1\}^{*}$ is the set of all strings (including the empty string). For positive integers $n$ and $m$ such that $n \leq 2^{m}-1,[n]_{m}$ is the $m$-bit binary representation of $n$. For a bit string $x$ and a positive integer $n$ such that $|x| \geq n$, first $(n, x)$ and last $(n, x)$ denote the first $n$ bits of $x$ and the last $n$ bits of $x$, respectively. For a positive integer $n, 0^{n}$ and $1^{n}$ denote the $n$-times repetition of 0 and 1 , respectively.

Blockciphers and function families. The blockcipher (permutation family) is a function $E: \mathcal{K} \times\{0,1\}^{n} \rightarrow\{0,1\}^{n}$, where, for any $K \in \mathcal{K}, E(K, \cdot)=E_{K}(\cdot)$ is a permutation on $\{0,1\}^{n}$. The positive integer $n$ is the block length and an $n$-bit string is called a block. If $\mathcal{K}=\{0,1\}^{k}$, then $k$ is the key length.

The PRP notion for blockciphers was introduced in [18] and later made concrete in [3]. Let $\operatorname{Perm}(n)$ denote the set of all permutations on $\{0,1\}^{n}$. This set can be regarded as a blockcipher by considering that each permutation is specified by a unique string. $P$ is a random permutation if $P \stackrel{R}{\leftarrow} \operatorname{Perm}(n)$. An adversary is a probabilistic algorithm (a program) with access to one or more oracles. Let $A$ be an adversary with access to an oracle, either the encryption oracle $E_{K}(\cdot)$ or a random permutation oracle $P(\cdot)$, and returns a bit. We say $A$ is a PRP-adversary for $E$, and we define

$$
\mathbf{A d v}_{E}^{\text {prp }}(A) \stackrel{\text { def }}{=}\left|\operatorname{Pr}\left(K \stackrel{R}{\leftarrow} \mathcal{K}: A^{E_{K}(\cdot)}=1\right)-\operatorname{Pr}\left(P \stackrel{R}{\leftarrow} \operatorname{Perm}(n): A^{P(\cdot)}=1\right)\right| .
$$

Similarly, the function family is a function $F: \mathcal{K} \times\{0,1\}^{m} \rightarrow\{0,1\}^{n}$, where, for any $K \in \mathcal{K}, F(K, \cdot)=F_{K}(\cdot)$ is a function from $\{0,1\}^{m}$ to $\{0,1\}^{n}$. Let $\operatorname{Func}(m, n)$ denote the set of all functions from $\{0,1\}^{m}$ to $\{0,1\}^{n}$. This set can be regarded as a function family by considering that each function in $\operatorname{Func}(m, n)$ is specified by a unique string. $R$ is a random function if $R \stackrel{R}{\leftarrow} \operatorname{Func}(m, n)$. Let $A$ be an adversary with access to an oracle, either $F_{K}(\cdot)$ or a random function oracle $R(\cdot)$, and returns a bit. We say $A$ is a $P R F$-adversary for $F$, and we define

$$
\mathbf{A d v}_{F}^{\text {prf }}(A) \stackrel{\text { def }}{=}\left|\operatorname{Pr}\left(K \stackrel{R}{\leftarrow} \mathcal{K}: A^{F_{K}(\cdot)}=1\right)-\operatorname{Pr}\left(R \stackrel{R}{\leftarrow} \operatorname{Func}(m, n): A^{R(\cdot)}=1\right)\right| .
$$

For an adversary $A, A$ 's running time is denoted by time $(A)$. The running time is its actual running time (relative to some fixed RAM model of computation) and its description size (relative to some standard encoding of algorithms). The details of the big- $O$ notation for the running time reference depend on the RAM model and the choice of encoding.

The frame, nonce, and counter. The modes described in this paper take a positive integer $w$ as a parameter, and it is called a frame width. For fixed positive integer $w$ (say, $w=2^{8}$ ), a $w$-block string is called a frame. Throughout this paper, we assume $w \geq 1$. A nonce $N$ is a bit string, where for each pair of key and plaintext, it is used only once. The length of the nonce is denoted by $\ell_{\text {nonce }}$, and it is at most the block length. We also use an $n$-bit string called a counter, ctr. This value is initialized based on the value of the nonce, then it is incremented after each blockcipher invocations. The function for increment is 


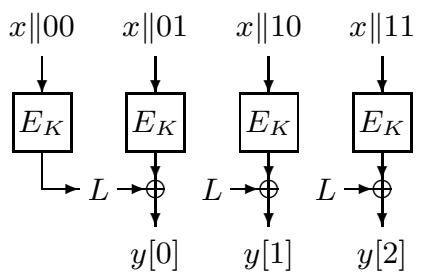

Fig. 1. Example illustration of $F$. In this example, $w=3, \omega=1+\left\lfloor\log _{2} w\right\rfloor=2$, and $F:\{0,1\}^{k} \times\{0,1\}^{n-2} \rightarrow\left(\{0,1\}^{n}\right)^{3}$ where $F_{K}(x)=(y[0], y[1], y[2])$. Here $x \in\{0,1\}^{n-2}$, $y[0]=L \oplus E_{K}(x \| 01), y[1]=L \oplus E_{K}(x \| 10), y[2]=L \oplus E_{K}(x \| 11)$, where $L=E_{K}(x \| 00)$.

denoted by inc $(\cdot)$. It takes an $n$-bit string $x$ (possibly a counter) and returns the incremented $x$. We assume $\operatorname{inc}(x)=x+1 \bmod 2^{n}$, but other implementations also work, e.g., with LFSRs if $x \neq 0^{n}$. For $i>0$, inc ${ }^{i}$ (ctr) means ctr is incremented for $i$ times. Since the value is initialized based on the value of the nonce, there is no need to maintain this value across the massages.

\section{The Basic Tool: A New Pseudorandom Function $F$}

In this section, we define a new function family $F$. It takes two parameters, a blockcipher, and a frame width.

Fix the blockcipher $E:\{0,1\}^{k} \times\{0,1\}^{n} \rightarrow\{0,1\}^{n}$, and the frame width $w$. Define $\omega=1+\left\lfloor\log _{2} w\right\rfloor$, i.e., we need $\omega$ bits to represent $w$. Now we define the function family $F:\{0,1\}^{k} \times\{0,1\}^{n-\omega} \rightarrow\left(\{0,1\}^{n}\right)^{w}$ as $F_{K}(x)=(y[0], \ldots, y[w-$ $1])$, where $y[i]=L \oplus E_{K}\left(\operatorname{inc}^{i+1}\left(x \|[0]_{\omega}\right)\right)$ for $i=0, \ldots, w-1$ and $L=E_{K}\left(x \|[0]_{\omega}\right)$.

We call $L$ a mask. See Figure 1 for an example.

We have the following information theoretic result on $F$.

Theorem 1. Let Perm $(n)$ and $w$ be the parameters for F. Let $A$ be a PRFadversary for $F$ making at most $q$ oracle queries. Then

$$
\mathbf{A d v}_{F}^{\operatorname{prf}}(A) \leq \frac{(w+1)^{4} q^{3}}{2^{2 n+1}}+\frac{w(w+1) q}{2^{n+1}}
$$

Notice that $w$ is a constant and the security bound of Theorem 1 is "beyond the birthday bound." Also, if we set $\sigma=q w$ (i.e., the total number of blocks that the adversary obtains) and measure the security bound in terms of $\sigma$, we have $\mathbf{A d v}_{F}^{\operatorname{prf}}(A) \leq w \sigma^{3} / 2^{2 n-3}+w \sigma / 2^{n}$, since $1+w \leq 2 w$.

The following definition is useful in proving Theorem 1 .

Definition 1. Let $x=\left(x_{0}, \ldots, x_{q-1}\right) \in\left(\{0,1\}^{n-\omega}\right)^{q}$ be an arbitrary $(n-\omega) q$ bit string. We say that " $x$ is distinct," if $x_{i} \neq x_{j}$ for $0 \leq i<j \leq q-1$. Similarly, let $Y=\left(Y_{0}, \ldots, Y_{q-1}\right) \in\left(\{0,1\}^{n w}\right)^{q}$ be an arbitrary nqw-bit string, where $Y_{i}=\left(y_{i}[0], \ldots, y_{i}[w-1]\right) \in\left(\{0,1\}^{n}\right)^{w}$ for $0 \leq i \leq q-1$. We say that " $Y$ is non-zero-distinct," if there is no equal bit strings in $\left\{0^{n}, y_{i}[0], \ldots, y_{i}[w-1]\right\}$ for any $i$ s.t. $0 \leq i \leq q-1$. 
Note that $0^{n}$ is included in the definition for " $Y$ is non-zero-distinct." Suppose that $F_{K}\left(x_{i}\right)=\left(y_{i}[0], \ldots, y_{i}[w-1]\right)$. Then we always have $y_{i}[j] \neq 0^{n}$, and we also see that $y_{i}[j] \neq y_{i}\left[j^{\prime}\right]$ for $j \neq j^{\prime}$. We allow, for example, $y_{i}[j]=y_{i^{\prime}}\left[j^{\prime}\right]$ for $i \neq i^{\prime}$. Intuitively, Definition 1 is the set of possible input-output pairs, and for these pairs the following lemma, which will be used in the proof of Theorem 1, shows that the distribution is close to uniform. This is the crucial observation for the security improvement. There are no collisions in "one frame," but the collision occurs across the frames.

Lemma 1. Let $x=\left(x_{0}, \ldots, x_{q-1}\right) \in\left(\{0,1\}^{n-\omega}\right)^{q}$ and $Y=\left(Y_{0}, \ldots, Y_{q-1}\right) \in$ $\left(\{0,1\}^{n w}\right)^{q}$ be arbitrarily fixed bit strings, where $x$ is distinct and $Y$ is non-zerodistinct. Then

$$
\frac{p_{F}}{p_{R}} \geq 1-\frac{q^{3}(w+1)^{4}}{2^{2 n+1}},
$$

where $p_{F} \stackrel{\text { def }}{=} \operatorname{Pr}\left(P \stackrel{R}{\leftarrow} \operatorname{Perm}(n): F_{P}\left(x_{i}\right)=Y_{i}\right.$ for $\left.0 \leq i \leq q-1\right)$ and $p_{R} \stackrel{\text { def }}{=}$ $\operatorname{Pr}\left(R \stackrel{R}{\leftarrow}\right.$ Func $(n-\omega, n w): R\left(x_{i}\right)=Y_{i}$ for $\left.0 \leq i \leq q-1\right)$.

The proof is based on the counting argument.

Proof (of Lemma 1). We first count the number of $P \in \operatorname{Perm}(n)$ which satisfies $F_{P}\left(x_{i}\right)=Y_{i}$ for $0 \leq i \leq q-1$. Let $L_{0}, \ldots, L_{q-1}$ be $n$-bit variables. Then the number of $L_{0}, \ldots, L_{q-1}$ which satisfy $\left\{L_{i}, L_{i} \oplus y_{i}[0], \ldots, L_{i} \oplus y_{i}[w-1]\right\} \cap\left\{L_{j}, L_{j} \oplus\right.$ $\left.y_{j}[0], \ldots, L_{j} \oplus y_{j}[w-1]\right\}=\emptyset$ for any $0 \leq i<j \leq q-1$ is at least $\prod_{0 \leq i \leq q-1}\left(2^{n}-\right.$ $\left.i(w+1)^{2}\right)$, since there are $2^{n}$ possibilities for $L_{0}$, and once $L_{0}, \ldots, L_{i-1}$ are fixed, we have at least $2^{n}-i(w+1)^{2}$ possibilities for $L_{i}$. If we set $L_{i}=P\left(x_{i} \|[0]_{\omega}\right)$, then it is possible to set $P\left(\operatorname{inc}\left(x_{i} \|[0]_{\omega}\right)\right)=L_{i} \oplus y_{i}[0], \ldots, P\left(\right.$ inc $\left.^{w}\left(x_{i} \|[0]_{\omega}\right)\right)=$ $L_{i} \oplus y_{i}[w-1]$ uniquely. We have fixed $q(w+1)$ input-output pairs of $P$, and the remaining $2^{n}-q(w+1)$ entries can be any value. Therefore, the number of $P \in \operatorname{Perm}(n)$ which satisfies $F_{P}\left(x_{i}\right)=Y_{i}$ for $0 \leq i \leq q-1$ is at least $\left(2^{n}-q(w+1)\right) ! \prod_{0 \leq i \leq q-1}\left(2^{n}-i(w+1)^{2}\right)$.

Then, the left hand side of (1) is at least

$$
\begin{aligned}
\frac{\left(2^{n}\right)^{q w}\left(2^{n}-q(w+1)\right) ! \prod_{0 \leq i \leq q-1}\left(2^{n}-i(w+1)^{2}\right)}{\left(2^{n}\right) !} & \\
& \geq \prod_{0 \leq i \leq q-1} \frac{1-\frac{i(w+1)^{2}}{2^{n}}}{\left(1-\frac{i(w+1)}{2^{n}}\right)\left(1-\frac{i(w+1)+1}{2^{n}}\right) \cdots\left(1-\frac{i(w+1)+w}{2^{n}}\right)} \\
& \geq \prod_{0 \leq i \leq q-1}\left(1-\frac{i(w+1)^{2}}{2^{n}}\right)\left(1+\frac{i(w+1)^{2}}{2^{n}}+\frac{w(w+1)}{2^{n+1}}\right) .
\end{aligned}
$$

We have used the fact that $(1-\alpha)^{-1} \geq 1+\alpha$ for $|\alpha|<1$, and the right hand side of (11) is given by simplifying (2).

The proof of Theorem 1 uses Lemma 1, and is given in Appendix $\mathrm{A}$. 


\section{A Relaxed Version $\boldsymbol{F}^{+}$}

In $F$, if the input is $x$, then the mask is always generated with $x \|[0]_{\omega}$. In this section, we present a slightly relaxed version of $F$, called $F^{+}$, which removes this restriction. Similarly to $F, F^{+}$takes two parameters, a blockcipher $E$ : $\{0,1\}^{k} \times\{0,1\}^{n} \rightarrow\{0,1\}^{n}$, and a frame width $w$.

Now the function family $F^{+}:\{0,1\}^{k} \times\{0,1\}^{n} \rightarrow\left(\{0,1\}^{n}\right)^{w}$ is defined as $F_{K}^{+}(x)=(y[0], \ldots, y[w-1])$, where $y[i]=L \oplus E_{K}\left(\right.$ inc $\left.^{i+1}(x)\right)$ for $i=0, \ldots, w-1$ and $L=E_{K}(x)$.

Observe that $F^{+}$takes $n$-bit $x$ as input, and the mask is generated with $x$. Also, it is not hard to show that $F^{+}$is a good PRF as long as there is no collision in the input to $E$.

Let $A$ be an adversary that makes at most $q$ oracle queries and let $x_{i} \in\{0,1\}^{n}$ denote $A$ 's $i$-th query. Define $X_{i}=\left\{x_{i}, \operatorname{inc}\left(x_{i}\right), \operatorname{inc}^{2}\left(x_{i}\right), \ldots, \operatorname{inc}^{w}\left(x_{i}\right)\right\}$, i.e., $X_{i}$ is the set of input to $E$ in the $i$-th query. We say that $A$ is input-respecting if $X_{i} \cap X_{j}=\emptyset$ for any $0 \leq i<j \leq q-1$, regardless of oracle responses and regardless of $A$ 's internal coins.

We have the following information theoretic result on $F^{+}$.

Corollary 1. Let Perm $(n)$ and $w$ be the parameters for $F^{+}$. Let $A$ be a PRFadversary for $F^{+}$making at most $q$ oracle queries, where $A$ is input-respecting. Then

$$
\mathbf{A d v}_{F^{+}}^{\text {prf }}(A) \leq \frac{(w+1)^{4} q^{3}}{2^{2 n+1}}+\frac{w(w+1) q}{2^{n+1}} .
$$

The proof is almost the same as that of Theorem 1, and omitted.

\section{CENC: Cipher-Based ENCryption}

In this section, we propose a new (nonce-based) encryption scheme, CENC. It takes three parameters, a blockcipher, a nonce length, and a frame width.

Fix the blockcipher $E:\{0,1\}^{k} \times\{0,1\}^{n} \rightarrow\{0,1\}^{n}$, the nonce length $\ell_{\text {nonce }}$ and the frame width $w$, where $1 \leq \ell_{\text {nonce }}<n$. CENC consists of two algorithms, the encryption algorithm (CENC.Enc) and the decryption algorithm (CENC.Dec). Both algorithms internally use the keystream generation algorithm (CENC.KSGen). These algorithms are defined in Figure2 A picture illustrating CENC.KSGen is given in Figure 3 ,

CENC.Enc has the following syntax. CENC.Enc : Key $\times$ Nonce $\times$ Plaintext $\rightarrow$ Ciphertext, where Key is $\{0,1\}^{k}$, Nonce is $\{0,1\}^{\ell_{\text {nonce }}}$, and Plaintext and Ciphertext are $\left\{M \in\{0,1\}^{*}|| M \mid \leq n 2^{\ell_{\max }}\right\}$, i.e., the set of bit strings at most $\ell_{\max }$ blocks, where $\ell_{\max }$ is the largest integer satisfying $\ell_{\max } \leq w\left(2^{n-\ell_{\text {nonce }}}-1\right) /(w+$ $1)$. It takes the key $K$, the nonce $N$, and the plaintext $M$ to return the ciphertext $C$. We write $C \leftarrow \operatorname{CENC.Enc}_{K}(N, M)$. The decryption algorithm CENC.Dec : Key $\times$ Nonce $\times$ Ciphertext $\rightarrow$ Plaintext takes $K, N, C$ to return $M$. We write $M \leftarrow \operatorname{CENC}^{-D} \operatorname{Dec}_{K}(N, C)$. For any $K, N$, and $M$, we have $M \leftarrow$

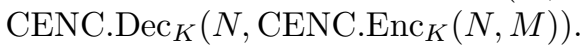




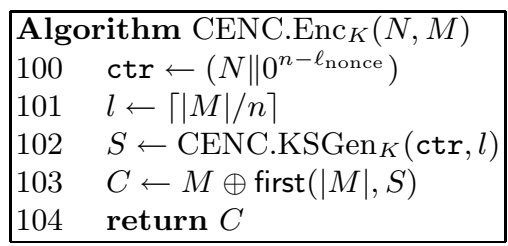

\begin{tabular}{|c|c|}
\hline & rithm CENC.Dec CE$_{K}(N, C)$ \\
\hline 200 & $\operatorname{ctr} \leftarrow\left(N \| 0^{n-\ell_{\text {nonce }}}\right)$ \\
\hline 201 & $l \leftarrow\lceil|C| / n\rceil$ \\
\hline 202 & $S \leftarrow$ CENC.KSGen $_{K}(\operatorname{ctr}, l)$ \\
\hline 203 & $M \leftarrow C \oplus$ first $(|C|, S)$ \\
\hline 204 & return $M$ \\
\hline
\end{tabular}

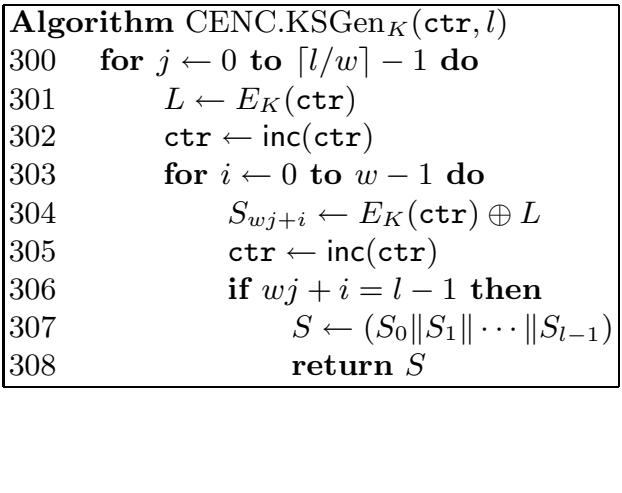

Fig. 2. Definition of the encryption algorithm CENC.Enc (left top), the decryption algorithm CENC.Dec (left bottom), and the keystream generation algorithm CENC.KSGen (right), which is used in both encryption and decryption

CENC.Enc and CENC.Dec call CENC.SKGen to generate the keystream of required length, where the length is in blocks. The encryption (resp. decryption) is just the xor of the plaintext (resp. ciphertext) and the keystream.

The keystream generation algorithm, CENC.KSGen, takes $K$, the initial counter value ctr, and a non-negative integer $l$. The output is a keystream $S$, where the length of $S$ is $l$ blocks. We write $S \leftarrow \operatorname{CENC.KSGen}_{K}(\operatorname{ctr}, l)$.

In CENC.KSGen, we first generate an $n$-bit mask, $L$. $\lceil l / w\rceil$ is the number of frames, incomplete frame counts as one frame. We see that $\lceil l / w\rceil$ masks are generated in line 301. For each mask, $w$ blocks of the keystream are generated in line 304 (except for the last frame, as the last frame may have fewer than $w$ blocks). If $l$ blocks of keystream are generated in line 306, the resulting $S$ is returned in line 308. Observe that the blockcipher is invoked for $l+\lceil l / w\rceil$ times, since we generate $\lceil l / w\rceil$ masks and we have $l$ blocks of keystream, where each block of keystream requires one blockcipher invocation.

Discussion and default parameters. CENC takes the blockcipher $E:\{0,1\}^{k} \times$ $\{0,1\}^{n} \rightarrow\{0,1\}^{n}$, the nonce length $\ell_{\text {nonce }}\left(1 \leq \ell_{\text {nonce }}<n\right)$ and the frame width $w$, as the parameters. With these parameters, CENC can encrypt at most $2^{\ell_{\text {nonce }}}$ plaintexts, and the maximum length of the plaintext is $\ell_{\max }$ blocks. Note that $\ell_{\max }$ is derived by solving $\ell_{\max }+\left\lceil\ell_{\max } / w\right\rceil \leq 2^{n-\ell_{\text {nonce }}}$ in $\ell_{\max }$, and in general, the bound on $\ell_{\max }$ is $\ell_{\max } \leq 2^{n-\ell_{\text {nonce }}-1}$ since $\left[\ell_{\max } / w\right] \leq \ell_{\max }$. As we will present in Section 6, the security bound of CENC is $(w+1)^{4} \hat{\sigma}^{3} / w^{3} 2^{2 n+1}+(w+1) \hat{\sigma} / 2^{n+1}$, where $\hat{\sigma}$ is (roughly) the total number of blocks processed by one key.

Our default parameters are, $E$ is any blockcipher such that $n \geq 128, \ell_{\text {nonce }}=$ $n / 2$, and $w=2^{8}=256$. For example, if we use the AES, CENC can encrypt at most $2^{64}$ plaintexts, the maximum length of the plaintext is $2^{63}$ blocks $\left(2^{37}\right.$ GBytes), and the security bound is $\hat{\sigma}^{3} / 2^{248}+\hat{\sigma} / 2^{121}$ (we used $(w+1)^{4} / w^{3}<$ $261<2^{9}$ ), thus $\hat{\sigma}$ should be sufficiently smaller that $2^{82}$ blocks $\left(2^{56}\right.$ GBytes). 


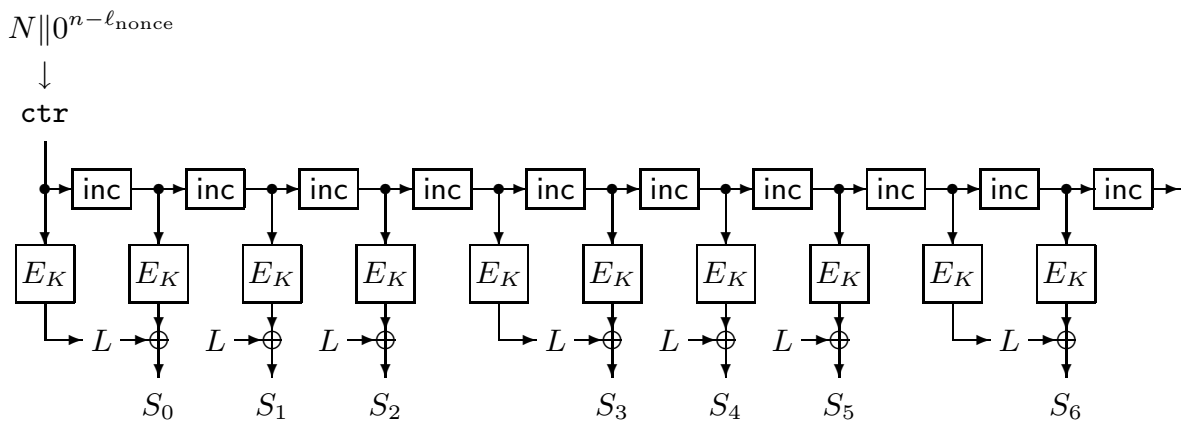

Fig. 3. Illustration of the keystream generation algorithm. This example uses $w=3$ and outputs $l=7$ blocks of keystream $S=\left(S_{0}, \ldots, S_{6}\right)$. This $S$ is used in both encryption and decryption. The mask $L$ is updated after generating $w$ blocks of keystream. The counter ctr is incremented for $l+\lceil l / w\rceil=10$ times, and there are 10 blockcipher invocations.

The frame width, $w$, should be large enough so that we can implement CENC efficiently. On the other hand, it affects the security bound. We chose $w=2^{8}=$ 256, which implies 256 blocks of keystream are generated with 257 blockcipher invocations, thus the cost is about $0.4 \%$ compared to CTR mode. We see that the efficiency loss is very small in both software and hardware. Also, the security bound is low enough with this value of $w$. We do not recommend $w>2^{8}$ (when $n=128$ ) because of the security loss.

64-bit blockciphers. We do not claim that CENC is generally useful for $n=64$, since there are restrictions on the nonce length (thus the number of plaintexts), and the plaintext length.

For example, if we use Triple-DES and $\left(\ell_{\text {nonce }}, w\right)=(32,256)$, CENC can encrypt at most $2^{32}$ plaintexts, and the maximum length of the plaintext is $2^{31}$ blocks (16GBytes), which may not be enough for general applications (still, it is comparable to CTR mode). In this case, the security bound is $\hat{\sigma}^{3} / 2^{120}+\hat{\sigma} / 2^{57}$, which implies $\hat{\sigma}$ should be sufficiently smaller that $2^{40}$ blocks $\left(2^{13}\right.$ GBytes $)$.

The limitations of the nonce length and the plaintext length can be removed if we use a counter (instead of a nonce) that is maintained across the plaintexts. This "counter version of CENC" is more suitable for 64-bit blockciphers.

\section{Security of CENC}

CENC is a symmetric encryption scheme. Before showing the security results on CENC, we first formally define what we mean by symmetric encryption schemes, and what we mean by such schemes to be secure.

Symmetric encryption schemes. A (nonce-based) symmetric encryption scheme is a pair of algorithms $\mathcal{S E}=(\mathcal{E}, \mathcal{D})$ where $\mathcal{E}$ is a deterministic encryption algorithm $\mathcal{E}:$ Key $\times$ Nonce $\times$ Plaintext $\rightarrow$ Ciphertext and $\mathcal{D}$ is a deterministic 
decryption algorithm $\mathcal{D}:$ Key $\times$ Nonce $\times$ Ciphertext $\rightarrow$ Plaintext. The key space Key is a set of keys, and is a nonempty set having a distribution (the uniform distribution when the set is finite). The nonce space Nonce, the plaintext space Plaintext, and the ciphertext space Ciphertext are nonempty sets of strings. We write $\mathcal{E}_{K}(N, M)$ for $\mathcal{E}(K, N, M)$ and $\mathcal{D}_{K}(N, C)$ for $\mathcal{D}(K, N, C)$. We require that $\mathcal{D}_{K}\left(N, \mathcal{E}_{K}(N, M)\right)=M$ for all $K \in \mathrm{Key}, N \in$ Nonce and $M \in$ Plaintext.

Nonce-respecting adversary. Let $A$ be an adversary with access to an encryption oracle $\mathcal{E}_{K}(\cdot, \cdot)$. This oracle, on input $(N, M)$, returns $C \leftarrow \mathcal{E}_{K}(N, M)$. Let $\left(N_{0}, M_{0}\right), \ldots,\left(N_{q-1}, M_{q-1}\right)$ denote its oracle queries. The adversary is said to be nonce-respecting if $N_{0}, \ldots, N_{q-1}$ are always distinct, regardless of oracle responses and regardless of $A$ 's internal coins.

Privacy of symmetric encryption schemes. We adopt the strong notion of privacy for nonce-based encryption schemes from [23]. This notion, which we call indistinguishability from random strings, provably implies the more standard notions given in [1].

Let $A$ be an adversary with access to an oracle, either the encryption oracle $\mathcal{E}_{K}(\cdot, \cdot)$ or $\mathcal{R}(\cdot, \cdot)$, and returns a bit. The $\mathcal{R}(\cdot, \cdot)$ oracle, on input $(N, M)$, returns a random string of length $\left|\mathcal{E}_{K}(N, M)\right|$. We say that $A$ is a PRIV-adversary for $\mathcal{S E}$. We assume that any PRIV-adversary is nonce-respecting. The advantage of PRIV-adversary $A$ for $\mathcal{S E}=(\mathcal{E}, \mathcal{D})$ having key space Key is

$$
\operatorname{Adv}_{\mathcal{S E}}^{\text {priv }}(A) \stackrel{\text { def }}{=} \mid \operatorname{Pr}\left(K \stackrel{R}{\leftarrow} \text { Key }: A^{\mathcal{E}_{K}(\cdot, \cdot)}=1\right)-\operatorname{Pr}\left(A^{\mathcal{R}(\cdot, \cdot)}=1\right) \mid .
$$

Security results on $C E N C$. Let $A$ be a nonce-respecting PRIV-adversary for CENC, and assume that $A$ makes at most $q$ oracle queries, and the total length of these queries is at most $\sigma$ blocks, where "the total length of queries" is defined as follows: if $A$ makes $q$ queries $\left(N_{0}, M_{0}\right), \ldots,\left(N_{q-1}, M_{q-1}\right)$, then the total length of queries is $\sigma=\left\lceil\left|M_{0}\right| / n\right\rceil+\cdots+\left\lceil\left|M_{q-1}\right| / n\right\rceil$, i.e, the total number of blocks of plaintexts. We have the following information theoretic result.

Theorem 2. Let Perm $(n), \ell_{\text {nonce, }}$ and $w$ be the parameters for CENC. Let $A$ be a nonce-respecting PRIV-adversary for CENC making at most $q$ oracle queries, and the total length of these queries is at most $\sigma$ blocks. Then

$$
\mathbf{A d v}_{\mathrm{CENC}}^{\mathrm{priv}}(A) \leq \frac{(w+1)^{4} \hat{\sigma}^{3}}{w^{3} 2^{2 n+1}}+\frac{(w+1) \hat{\sigma}}{2^{n+1}}
$$

where $\hat{\sigma}=\sigma+q w$.

If we use the rough inequality of $w+1 \leq 2 w$, then we have the simpler form, $\operatorname{Adv}_{\mathrm{CENC}}^{\text {priv }}(A) \leq w \hat{\sigma}^{3} / 2^{2 n-3}+w \hat{\sigma} / 2^{n}$.

The proof of Theorem 2 is based on the contradiction argument. If there exists a nonce-respecting PRIV-adversary $A$ such that $\mathbf{A d v}_{\text {CENC }}^{\text {priv }}(A)$ is larger than the right hand side of (3), then we can construct an input-respecting PRF-adversary $B$ for $F^{+}$which contradicts Corollary 1 , The proof is given in Appendix B.

Given Theorem 2, we have the following complexity theoretic result. 


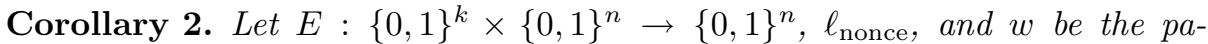
rameters for $C E N C$. Let $A$ be a nonce-respecting PRIV-adversary for $C E N C$ making at most $q$ oracle queries, and the total length of these queries is at most $\sigma$ blocks. Then there is a PRP-adversary $B$ for $E$ making at most $(w+1) \hat{\sigma} / w$ oracle queries, time $(B)=\operatorname{time}(A)+O(n \hat{\sigma} w)$, and $\mathbf{A d v}_{E}^{\text {prp }}(B) \geq \mathbf{A d v}_{\mathrm{CENC}}^{\text {priv }}(A)-$ $w \hat{\sigma}^{3} / 2^{2 n-3}-w \hat{\sigma} / 2^{n}$, where $\hat{\sigma}=\sigma+q w$.

The proof of Corollary 2 is given in [1].

\section{CHM: CENC with Hash-Based MAC}

In this section, we present a new (nonce-based) authenticated-encryption with associated-data (AEAD) scheme, CHM. It takes six parameters, a blockcipher, a nonce length, a tag length, a frame width, and two constants.

Fix the blockcipher $E:\{0,1\}^{k} \times\{0,1\}^{n} \rightarrow\{0,1\}^{n}$, the nonce length $\ell_{\text {nonce }}$, the tag length $\tau$, the frame width $w$, and two $n$-bit constants const $t_{0}$ and const . $_{1}$. We require that $1 \leq \ell_{\text {nonce }}<n, 1 \leq \tau \leq n$, const $_{0} \neq$ const $_{1}$, and first $\left(1\right.$, const and $\left._{0}\right)=$

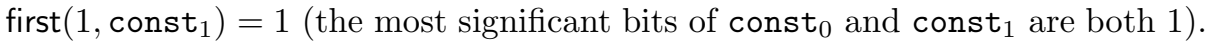

CHM consists of two algorithms, the encryption algorithm (CHM.Enc) and the decryption algorithm (CHM.Dec). These algorithms are defined in Figure 4 Both algorithms use the keystream generation algorithm (CHM.KSGen) and a hash function (CHM.Hash). CHM.KSGen is equivalent to CENC.KSGen defined in Figure 2, and the hash function CHM.Hash is defined in Figure 5,

The syntax of the encryption algorithm is CHM.Enc : Key $\times$ Nonce $\times$ Header $\times$ Plaintext $\rightarrow$ Ciphertext $\times$ Tag, where the key space Key is $\{0,1\}^{k}$, the nonce space Nonce is $\{0,1\}^{\ell_{\text {nonce }}}$, and the header space Header is $\{0,1\}^{*}$. The plaintext space Plaintext and ciphertext space Ciphertext are $\left\{M \in\{0,1\}^{*}|| M \mid \leq n 2^{\ell_{\max }}\right\}$, where $\ell_{\max }$ is the largest integer satisfying $\ell_{\max } \leq w\left(2^{n-\ell_{\text {nonce }}-1}-1\right) /(w+1)-1$. The tag space Tag is $\{0,1\}^{\tau}$. It takes the key $K$, the nonce $N$, the header $H$, and the plaintext $M$ to return the ciphertext $C$ and the tag $T$. We write $(C, T) \leftarrow \mathrm{CHM}^{\mathrm{Enn}} \mathrm{En}_{K}(N, H, M)$. The decryption algorithm CHM.Dec : Key $\times$ Nonce $\times$ Header $\times$ Ciphertext $\times$ Tag $\rightarrow$ Plaintext $\cup\{$ reject $\}$ takes $K, N, H, C$ and $T$ to return $M$ or a special symbol reject. We write $M \leftarrow \operatorname{CHM}^{-D_{e c}}(N, H, C, T)$

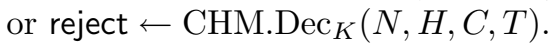

$\mathrm{CHM}$ is the natural combination of $\mathrm{CENC}$ and a universal hash functionbased MAC (Wegman-Carter MAC). As a universal hash function, we chose the standard polynomial-based hash, since it is efficient in both software and hardware, and it is well studied. The multiplication is done in the finite field $\operatorname{GF}\left(2^{n}\right)$ using a canonical polynomial to represent field elements. The suggested canonical polynomial is the lexicographically first polynomial among the irreducible polynomials of degree $n$ that have a minimum number of nonzero coefficients. For $n=128$ the indicated polynomial is $\mathrm{x}^{128}+\mathrm{x}^{7}+\mathrm{x}^{2}+\mathrm{x}+1$.

Discussion and default parameters. CHM takes six parameters, the blockcipher $E:\{0,1\}^{k} \times\{0,1\}^{n} \rightarrow\{0,1\}^{n}$, the nonce length $\ell_{\text {nonce }}$, the tag length $\tau$, the 


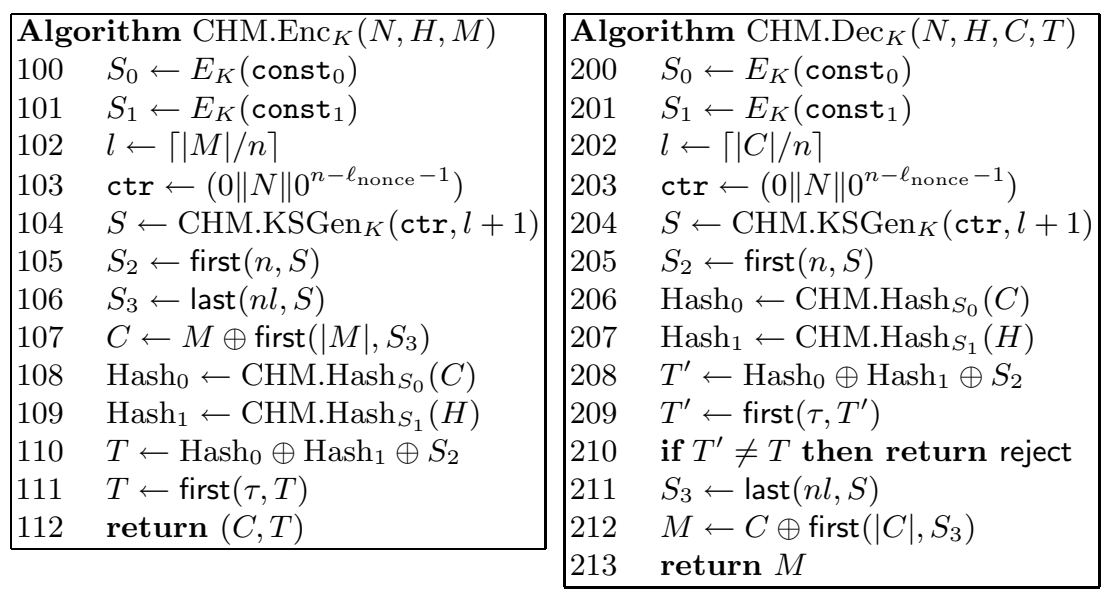

Fig. 4. Definition of the encryption algorithm CHM.Enc (left), and the decryption algorithm CHM.Dec (right). CHM.KSGen is equivalent to CENC.KSGen in Figure 2, and CHM.Hash is defined in Figure 5

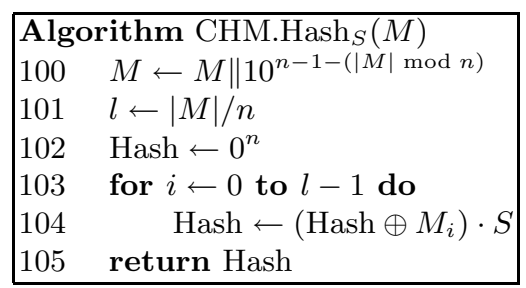

Fig. 5. Definition of CHM.Hash : $\{0,1\}^{n} \times\{0,1\}^{*} \rightarrow\{0,1\}^{n} . M_{i}$ is the $i$-th block of $M \| 10^{n-1-(|M| \bmod n)}$, i.e., $\left(M_{0}, \ldots, M_{l-1}\right)=M \| 10^{n-1-(|M| \bmod n)}$. Multiplication in line 104 is in $\mathrm{GF}\left(2^{n}\right)$.

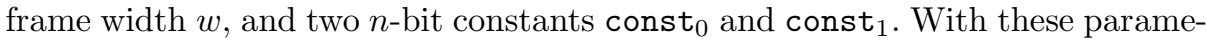
ters, CHM can encrypt at most $2^{\ell_{\text {nonce }}}$ plaintext-header pairs, and the maximum length of the plaintext is $\ell_{\max }$ blocks $\left(\ell_{\max }\right.$ is derived by solving $\ell_{\max }+1+\left\lceil\left(\ell_{\max }+\right.\right.$ $1) / w\rceil \leq 2^{n-\ell_{\text {nonce }}-1}$ in $\left.\ell_{\max }\right)$. As we will present in Section 8 the security bound of $\mathrm{CHM}$ is $(w+1)^{3} \tilde{\sigma}^{2} / w^{2} 2^{2 n-3}+(w+1)^{4} \tilde{\sigma}^{3} / w^{3} 2^{2 n+1}+1 / 2^{n}+(w+1) \tilde{\sigma} / 2^{n+1}$ for privacy, and $(w+1)^{3} \tilde{\sigma}^{2} / w^{2} 2^{2 n-3}+(w+1)^{4} \tilde{\sigma}^{3} / w^{3} 2^{2 n+1}+1 / 2^{n}+(w+1) \tilde{\sigma} / 2^{n+1}+$ $\left(1+H_{\max }+M_{\max }\right) / 2^{\tau}$ for authenticity, where $\tilde{\sigma}$ is (roughly) the total number of blocks processed by one key, $M_{\max }$ is the maximum block length of plaintexts, and $H_{\max }$ is the maximum block length of headers.

Our default parameters are, $E$ is any blockcipher such that $n \geq 128, \ell_{\text {nonce }}=$ $n / 2-1, \tau \geq 96, w=2^{8}=256$, const $_{0}=1^{n-1} \| 0$ and const $_{1}=1^{n}$.

With these parameters, if we use the AES, CHM can encrypt at most $2^{63}$ plaintexts-header pairs, and the maximum length of the plaintext is $2^{63}$ blocks $\left(2^{37}\right.$ GBytes $)$, and the security bounds are $\tilde{\sigma}^{3} / 2^{242}+\tilde{\sigma} / 2^{120}$ for privacy, and 
$\tilde{\sigma}^{3} / 2^{242}+\tilde{\sigma} / 2^{120}+\left(1+H_{\max }+M_{\max }\right) / 2^{\tau}$ for authenticity. This implies $\tilde{\sigma}$ should be sufficiently smaller that $2^{80}$ blocks $\left(2^{54}\right.$ GBytes $)$, and $H_{\max }$ and $M_{\max }$ should be small enough so that $\left(1+H_{\max }+M_{\max }\right) / 2^{\tau}$ is low enough.

\section{Security of CHM}

CHM is an authenticated-encryption with associated-data (AEAD) scheme. Before showing the security results on CHM, we first formally define what we mean by AEAD schemes, and what we mean by such schemes to be secure.

$A E A D$ schemes. A (nonce-based) authenticated-encryption with associated-data $(\mathrm{AEAD})$ scheme is a pair of algorithms $\mathcal{A} \mathcal{E}=(\mathcal{E}, \mathcal{D})$ where $\mathcal{E}$ is a deterministic encryption algorithm $\mathcal{E}:$ Key $\times$ Nonce $\times$ Header $\times$ Plaintext $\rightarrow$ Ciphertext $\times$ Tag and $\mathcal{D}$ is a deterministic decryption algorithm $\mathcal{D}: \operatorname{Key} \times$ Nonce $\times$ Header $\times$ Ciphertext $\times$ Tag $\rightarrow$ Plaintext $\cup\{$ reject $\}$. The key space Key is a set of keys. The nonce space Nonce and the header space Header (also called the space of associated data), the plaintext space Plaintext and the ciphertext space Ciphertext are nonempty sets of strings. (We note that there is a more general treatment where Ciphertext and Tag are not separated. See [7. We separate them for simplicity.) We write $\mathcal{E}_{K}(N, H, M)$ for $\mathcal{E}(K, N, H, M)$ and $\mathcal{D}_{K}(N, H, C, T)$ for $\mathcal{D}(K, N, H, C, T)$. We require that $\mathcal{D}_{K}\left(N, H, \mathcal{E}_{K}(N, H, M)\right)=M$ for all $K \in$ Key, $N \in$ Nonce, $H \in$ Header and $M \in$ Plaintext.

Privacy of AEAD schemes. We follow the security notion from [7. Let $A$ be an adversary with access to an oracle, either the encryption oracle $\mathcal{E}_{K}(\cdot, \cdot, \cdot)$ or $\mathcal{R}(\cdot, \cdot, \cdot)$, and returns a bit. The $\mathcal{R}(\cdot, \cdot, \cdot)$ oracle, on input $(N, H, M)$, returns a random string of length $\left|\mathcal{E}_{K}(N, H, M)\right|$. We say that $A$ is a PRIVadversary for $\mathcal{A} \mathcal{E}$. We assume that any PRIV-adversary is nonce-respecting (i.e., if $\left(N_{0}, H_{0}, M_{0}\right), \ldots,\left(N_{q-1}, H_{q-1}, M_{q-1}\right)$ is $A$ 's oracle queries, $N_{0}, \ldots, N_{q-1}$ are always distinct, regardless of oracle responses and regardless of $A$ 's internal coins). The advantage of PRIV-adversary $A$ for AEAD scheme $\mathcal{A E}=(\mathcal{E}, \mathcal{D})$ having key space Key is

$$
\mathbf{A d v}_{\mathcal{A}}^{\text {priv }}(A) \stackrel{\text { def }}{=} \mid \operatorname{Pr}\left(K \stackrel{R}{\leftarrow} \text { Key }: A^{\mathcal{E}_{K}(\cdot, \cdot, \cdot)}=1\right)-\operatorname{Pr}\left(A^{\mathcal{R}(\cdot, \cdot, \cdot)}=1\right) \mid .
$$

Authenticity of AEAD schemes. A notion of authenticity of ciphertext for AEAD schemes was formalized in 23|22] following [14|6|5]. This time, let $A$ be an adversary with access to an encryption oracle $\mathcal{E}_{K}(\cdot, \cdot, \cdot)$ and returns a tuple, $(N, H, C, T)$. This tuple is called a forgery attempt. We say that $A$ is an AUTHadversary for $\mathcal{A} \mathcal{E}$. We assume that any AUTH-adversary is nonce-respecting. (The condition is understood to apply only to the adversary's encryption oracle. Thus a nonce used in an encryption-oracle query may be used in a forgery attempt.) We say $A$ forges if $A$ returns $(N, H, C, T)$ such that $\mathcal{D}_{K}(N, H, C, T) \neq$ reject but $A$ did not make a query $(N, H, M)$ to $\mathcal{E}_{K}(\cdot, \cdot, \cdot)$ that resulted in a response $(C, T)$. That is, adversary $A$ may never return a forgery attempt 
$(N, H, C, T)$ such that the encryption oracle previously returned $(C, T)$ in response to a query $(N, H, M)$. Then the advantage of AUTH-adversary $A$ for AEAD scheme $\mathcal{A E}=(\mathcal{E}, \mathcal{D})$ having key space Key is

$$
\mathbf{A d v}_{\mathcal{A} \mathcal{E}}^{\text {auth }}(A) \stackrel{\text { def }}{=} \operatorname{Pr}\left(K \stackrel{R}{\leftarrow} \text { Key }: A^{\mathcal{E}_{K}(\cdot, \cdot, \cdot)} \text { forges }\right) .
$$

Privacy results on $C H M$. Let $A$ be a nonce-respecting PRIV-adversary for CHM, and assume that $A$ makes at most $q$ oracle queries, and the total plaintext length of these queries is at most $\sigma$ blocks, where "the total plaintext length of queries" is defined as follows: if $A$ makes queries $\left(N_{0}, H_{0}, M_{0}\right), \ldots,\left(N_{q-1}, H_{q-1}, M_{q-1}\right)$, then $\sigma=\left\lceil\left|M_{0}\right| / n\right\rceil+\cdots+\left\lceil\left|M_{q-1}\right| / n\right\rceil$, i.e., the total number of blocks of plaintexts. We have the following information theoretic result.

Theorem 3. Let $\operatorname{Perm}(n), \ell_{\text {nonce, }} \tau, w$, const $t_{0}$ and const $t_{1}$ be the parameters for CHM. Let $A$ be a nonce-respecting PRIV-adversary making at most $q$ oracle queries, and the total plaintext length of these queries is at most $\sigma$ blocks. Then

$$
\mathbf{A d v}_{\mathrm{CHM}}^{\text {priv }}(A) \leq \frac{(w+1)^{3} \tilde{\sigma}^{2}}{w^{2} 2^{2 n-3}}+\frac{(w+1)^{4} \tilde{\sigma}^{3}}{w^{3} 2^{2 n+1}}+\frac{1}{2^{n}}+\frac{(w+1) \tilde{\sigma}}{2^{n+1}},
$$

where $\tilde{\sigma}=\sigma+q(w+1)$.

Note that there is no restriction on the header length. If we use $w+1 \leq 2 w$, we have the simpler form, $\mathbf{A d v}_{\mathrm{CHM}}^{\text {priv }}(A) \leq w \tilde{\sigma}^{2} / 2^{2 n-6}+w \tilde{\sigma}^{3} / 2^{2 n-3}+1 / 2^{n}+w \tilde{\sigma} / 2^{n}$.

The proof of Theorem 3 is given in [11]. From Theorem 3 , we have the following complexity theoretic result.

Corollary 3. Let $E:\{0,1\}^{k} \times\{0,1\}^{n} \rightarrow\{0,1\}^{n}$, $\ell_{\text {nonce }} \tau, w$, const $t_{0}$ and const $_{1}$ be the parameters for CHM. Let $A$ be a nonce-respecting PRIV-adversary making at most $q$ oracle queries, and the total plaintext length of these queries is at most $\sigma$ blocks. Then there is a PRP-adversary $B$ for $E$ making at most $(w+1) \tilde{\sigma} / w$ oracle queries, time $(B)=$ time $(A)+O(n \tilde{\sigma} w)$, and $\mathbf{A d v}_{E}^{\text {prp }}(B) \geq$ $\mathbf{A d v}_{\mathrm{CHM}}^{\text {priv }}(A)-w \tilde{\sigma}^{2} / 2^{2 n-6}-w \tilde{\sigma}^{3} / 2^{2 n-3}-1 / 2^{n}-w \tilde{\sigma} / 2^{n}$, where $\tilde{\sigma}=\sigma+q(w+1)$.

The proof of Corollary 3 is given in [1].

Authenticity results on CHM. Let $A$ be an AUTH-adversary for CHM, and assume that $A$ makes at most $q$ oracle queries (including the final forgery attempt), the total plaintext length of these queries is at most $\sigma$ blocks, the maximum plaintext length of these queries is at most $M_{\max }$ blocks, and the maximum header length of these queries is at most $H_{\max }$ blocks. Here, if $A$ makes queries $\left(N_{0}, H_{0}, M_{0}\right), \ldots,\left(N_{q-2}, H_{q-2}, M_{q-2}\right)$, and returns the forgery attempt $\left(N^{*}, H^{*}, C^{*}, T^{*}\right)$, then $\sigma, M_{\max }$ and $H_{\max }$ are defined as

$$
\left\{\begin{array}{l}
\sigma \stackrel{\text { def }}{=}\left\lceil\left|M_{0}\right| / n\right\rceil+\cdots+\left\lceil\left|M_{q-2}\right| / n\right\rceil+\left\lceil\left|C^{*}\right| / n\right\rceil, \\
M_{\max } \stackrel{\text { def }}{=} \max \left\{\left\lceil\left|M_{0}\right| / n\right\rceil, \ldots,\left\lceil\left|M_{q-2}\right| / n\right\rceil,\left\lceil\left|C^{*}\right| / n\right\rceil\right\}, \\
H_{\max } \stackrel{\text { def }}{=} \max \left\{\left\lceil\left|H_{0}\right| / n\right\rceil, \ldots,\left\lceil\left|H_{q-2}\right| / n\right\rceil,\left\lceil\left|H^{*}\right| / n\right\rceil\right\} .
\end{array}\right.
$$

We say $A$ 's query resource is $\left(q, \sigma, M_{\max }, H_{\max }\right)$. We have the following information theoretic result. 
Theorem 4. Let Perm $(n), \ell_{\text {nonce}}, \tau, w$, const $t_{0}$ and const $t_{1}$ be the parameters for CHM. Let $A$ be a nonce-respecting AUTH-adversary whose query resource is $\left(q, \sigma, M_{\max }, H_{\max }\right)$. Then $\mathbf{A d v}_{\mathrm{CHM}}^{\text {auth }}(A)$ is at most

$$
\frac{(w+1)^{3} \tilde{\sigma}^{2}}{w^{2} 2^{2 n-3}}+\frac{(w+1)^{4} \tilde{\sigma}^{3}}{w^{3} 2^{2 n+1}}+\frac{1}{2^{n}}+\frac{(w+1) \tilde{\sigma}}{2^{n+1}}+\frac{1+H_{\max }+M_{\max }}{2^{\tau}},
$$

where $\tilde{\sigma}=\sigma+q(w+1)$.

If we use $w+1 \leq 2 w$, we have the simpler form, $\mathbf{A d v}_{\mathrm{CHM}}^{\text {auth }}(A) \leq w \tilde{\sigma}^{2} / 2^{2 n-6}+$ $w \tilde{\sigma}^{3} / 2^{2 n-3}+1 / 2^{n}+w \tilde{\sigma} / 2^{n}+\left(1+H_{\max }+M_{\max }\right) / 2^{\tau}$.

The proof of Theorem 4 is given in [1]. From Theorem 4 , we have the following complexity theoretic result.

Corollary 4. Let $E:\{0,1\}^{k} \times\{0,1\}^{n} \rightarrow\{0,1\}^{n}$, $\ell_{\text {nonce, }} \tau, w$, const $t_{0}$, and const $_{1}$ be the parameters for CHM. Let $A$ be a nonce-respecting AUTH-adversary whose query resource is $\left(q, \sigma, M_{\max }, H_{\max }\right)$. Then there is a PRP-adversary $B$ for $E$ making at most $(w+1) \tilde{\sigma} / w$ oracle queries, time $(B)=\operatorname{time}(A)+O(n \tilde{\sigma} w)$, and $\mathbf{A d v}_{E}^{\text {prp }}(B) \geq \mathbf{A d v}_{\mathrm{CHM}}^{\text {auth }}(A)-w \tilde{\sigma}^{2} / 2^{2 n-6}-w \tilde{\sigma}^{3} / 2^{2 n-3}-1 / 2^{n}+w \tilde{\sigma} / 2^{n}-$ $\left(1+H_{\max }+M_{\max }\right) / 2^{\tau}$, where $\tilde{\sigma}=\sigma+q(w+1)$.

\section{Discussions}

Counter-based versions. CENC and CHM use a nonce, and it is natural to consider their counter-based versions. Call them CENC-C and CHM-C, respectively. They use an $n$-bit counter maintained across the plaintexts (usually by the sender). The drawback is the difficulty of implementation and it is relatively harder to use them properly, which is the reason why we have concentrated on the nonce-based schemes. The advantage of CENC-C and CHM-C is that, the nonce length and the maximum plaintext length restrictions are removed, while the security is unchanged (further, non-adaptive version of PRP is enough for the security proofs). The restrictions only come from the security bound (instead of the schemes). Thus, if carefully implemented and properly used, these counter versions are suitable especially for 64-bit blockciphers

Tightness of the security bounds. For CTR mode, the security bound is tight up to a constant factor. However, for CENC and CHM (and the PRF F in Section 3), we do not know the tightness of our security bounds. The tightness is an open question. For example, if we take CENC, the bound is $O\left(w \hat{\sigma}^{3} / 2^{2 n}+w \hat{\sigma} / 2^{n}\right)$. The question is the existence of an adversary $A$ that breaks the privacy of CENC with about $\hat{\sigma}=2^{82}$ data (without breaking the pseudorandomness of the AES), or the proof that the security is better than the above. We conjecture that the bound of CENC can be improved to $O\left(w \hat{\sigma} / 2^{n}\right)$, possibly by using the technique from $[2]$.

${ }^{1}$ However, it is not possible to check the details of the proof of [2], since only a sketch is given. 


\section{Acknowledgement}

The author would like to thank Kazumaro Aoki, Fumihiko Sano, and Akashi Satoh for useful comments.

\section{References}

1. M. Bellare, A. Desai, E. Jokipii, and P. Rogaway. A concrete security treatment of symmetric encryption. Proceedings of The 38th Annual Symposium on Foundations of Computer Science, FOCS '97, pp. 394-405, IEEE, 1997.

2. M. Bellare, and R. Impagliazzo. A tool for obtaining tighter security analyses of pseudorandom function based constructions, with application to PRP $\rightarrow$ PRF convention. Cryptology ePrint Archive, Report 1999/024, Available at http://eprint.iacr.org/, 1999.

3. M. Bellare, J. Kilian, and P. Rogaway. The security of the cipher block chaining message authentication code. JCSS, vol. 61, no. 3, pp. 362-399, 2000. Earlier version in Advances in Cryptology - CRYPTO '94, LNCS 839, pp. 341-358, Springer-Verlag, 1994.

4. M. Bellare, T. Krovetz, and P. Rogaway. Luby-Rackoff backwards: Increasing security by making block ciphers non-invertible. Advances in CryptologyEUROCRYPT '98, LNCS 1403, pp. 266-280, Springer-Verlag, 1998.

5. M. Bellare, and C. Namprempre. Authenticated encryption: Relations among notions and analysis of the generic composition paradigm. Advances in CryptologyASIACRYPT 2000, LNCS 1976, pp. 531-545, Springer-Verlag, 2000.

6. M. Bellare, and P. Rogaway. Encode-then-encipher encryption: How to exploit nonces or redundancy in plaintexts for efficient cryptography. Advances in Cryptology-ASIACRYPT 2000, LNCS 1976, pp. 317-330, Springer-Verlag, 2000.

7. M. Bellare, P. Rogaway, and D. Wagner. The EAX mode of operation. Fast Software Encryption, FSE 2004, LNCS 3017, pp. 389-407, Springer-Verlag, 2004.

8. K. Claffy, G. Miller, and K. Thompson. The nature of the beast: Recent traffic measurements from an Internet backbone. Proceedings of INET '98. Available at http://www.caida.org/outreach/papers/1998/Inet98

9. D. Delov, C. Dwork, and M. Naor. Non-malleable cryptography. SIAM J. Comput., vol. 30, no. 2, pp. 391-437, 2000.

10. C. Hall, D. Wagner, J. Kelsey, and B. Schneier. Building PRFs from PRPs. Advances in Cryptology-CRYPTO '98, LNCS 1462, pp. 370-389, Springer-Verlag, 1998.

11. T. Iwata. New blockcipher modes of operation with beyond the birthday bound security. Full version of this paper. Available from the author, 2006.

12. J. Jonsson. On the Security of CTR + CBC-MAC. Selected Areas in Cryptography, 9th Annual Workshop (SAC 2002), LNCS 2595, pp. 76-93. Springer-Verlag, 2002.

13. C.S. Jutla. Encryption modes with almost free message integrity. Advances in Cryptology-EUROCRYPT 2001, LNCS 2045, pp. 529-544, Springer-Verlag, 2001.

14. J. Katz, and M. Yung. Unforgeable encryption and chosen ciphertext secure modes of operation. Fast Software Encryption, FSE 2000, LNCS 1978, pp. 284-299, Springer-Verlag, 2000.

15. T. Kohno, J. Viega, and D. Whiting. CWC: A high-performance conventional authenticated encryption mode. Fast Software Encryption, FSE 2004, LNCS 3017, pp. 408-426, Springer-Verlag, 2004. 
16. S. Lucks. The sum of PRPs is a secure PRF. Advances in CryptologyEUROCRYPT 2000, LNCS 1807, pp. 470-484, Springer-Verlag, 2000.

17. S. Lucks. The two-pass authenticated encryption faster than generic composition. Fast Software Encryption, FSE 2005, LNCS 3557, pp. 284-298, Springer-Verlag, 2005.

18. M. Luby and C. Rackoff. How to construct pseudorandom permutations from pseudorandom functions. SIAM J. Comput., vol. 17, no. 2, pp. 373-386, 1988.

19. D. McGrew, and J. Viega. The Galois/Counter mode of operation (GCM). Submission to NIST. Available at http://csrc.nist.gov/CryptoToolkit/modes/, 2004.

20. D. McGrew, and J. Viega. The security and performance of Galois/Counter mode of operation. Progress in Cryptology-INDOCRYPT 2004, LNCS 3348, pp. 343355, Springer-Verlag, 2004.

21. P. Rogaway. Nonce-based symmetric encryption. Fast Software Encryption, FSE 2004, LNCS 3017, pp. 348-358, Springer-Verlag, 2004.

22. P. Rogaway. Authenticated-encryption with associated-data. Proceedings of the ACM Conference on Computer and Communications Security, ACM CCS 2002, pp. 98-107, ACM, 2002.

23. P. Rogaway, M. Bellare, J. Black, and T. Krovetz. OCB: a block-cipher mode of operation for efficient authenticated encryption. ACM Trans. on Information System Security (TISSEC), vol. 6, no. 3, pp. 365-403, 2003. Earlier version in Proceedings of the eighth ACM Conference on Computer and Communications Security, ACM CCS 2001, pp. 196-205, ACM, 2001.

24. M.N. Wegman, and J.L. Carter. New hash functions and their use in authentication and set equality. JCSS, vol. 22, pp. 256-279, 1981.

25. D. Whiting, R. Housley, and N. Ferguson. Counter with CBC-MAC (CCM). Submission to NIST. Available at http://csrc.nist.gov/CryptoToolkit/modes/ 2002 .

\section{A Proof of Theorem 1}

Proof (of Theorem 11). Without loss of generality, we assume that $A$ makes exactly $q$ oracle queries and $A$ does not repeat an oracle query. Also, since $A$ is computationally unbounded, we assume that $A$ is deterministic. Now we can regard $A$ as a function $f_{A}:\left(\{0,1\}^{n w}\right)^{q} \rightarrow\{0,1\}$. To see this, let $Y=\left(Y_{0}, \ldots, Y_{q-1}\right)$ be an arbitrary $n q w$-bit string, where each $Y_{i}$ is $n w$ bits. The first query, $x_{0}$, is determined by $A$. If we return $Y_{i-1}$ as the answer for $x_{i-1}$, the next query $x_{i}$ is determined, and finally, if we return $Y_{q-1}$ as the answer for $x_{q-1}$, the output of $A$, either 0 or 1 , is determined. Therefore, the output of $A$ and the $q$ queries, $x_{0}, \ldots, x_{q-1}$, are all determined by fixing $Y$. Note that for any $Y$, the corresponding sequence of queries $x=\left(x_{0}, \ldots, x_{q-1}\right)$ is distinct. Let $\mathbf{v}_{\text {one }}=\left\{Y \in\left(\{0,1\}^{n w}\right)^{q} \mid f_{A}(Y)=1\right\}$, and $\mathbf{v}_{\text {dist }}=\left\{Y \in\left(\{0,1\}^{n w}\right)^{q} \mid\right.$ $Y$ is non-zero-distinct $\}$. Observe that $\left|\mathbf{v}_{\text {dist }}\right|=\left(\left(2^{n}-1\right)\left(2^{n}-2\right) \cdots\left(2^{n}-w\right)\right)^{q} \geq$ $2^{n w q}\left(1-q w(w+1) / 2^{n+1}\right)$, and therefore, we have

$$
\left|\mathbf{v}_{\text {one }} \cap \mathbf{v}_{\text {dist }}\right| \geq\left|\mathbf{v}_{\text {one }}\right|-2^{n w q} q w(w+1) / 2^{n+1} .
$$

Let $P_{R} \stackrel{\text { def }}{=} \operatorname{Pr}\left(R \stackrel{R}{\longleftarrow} \operatorname{Func}(n-\omega, n w): A^{R(\cdot)}=1\right)$. Then we have

$$
P_{R}=\sum_{Y \in \mathbf{v}_{\text {one }}} p_{R}=\frac{\left|\mathbf{v}_{\text {one }}\right|}{\left(2^{n w}\right)^{q}} .
$$




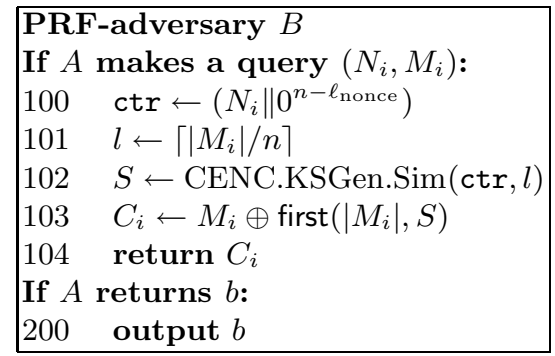

\begin{tabular}{|c|c|}
\hline Alg & rithm CENC.KSGen.Sin \\
\hline 300 & for $j \leftarrow 0$ to $\lceil l / w\rceil-1$ \\
\hline 301 & $Y_{j} \leftarrow \mathcal{O}(\mathrm{ctr})$ \\
\hline 302 & $\operatorname{ctr} \leftarrow \operatorname{inc}{ }^{w+1}(\operatorname{ctr})$ \\
\hline 303 & $Y \leftarrow\left(Y_{0}, \ldots, Y_{\lceil l / w\rceil-1}\right)$ \\
\hline 304 & $Y \leftarrow \operatorname{first}(n l, Y)$ \\
\hline 305 & return $Y$ \\
\hline
\end{tabular}

Fig. 6. The PRF-adversary $B$ for $F^{+}$based on the PRIV-adversary $A$ for CENC

On the other hand, let $P_{F} \stackrel{\text { def }}{=} \operatorname{Pr}\left(P \stackrel{R}{\leftarrow} \operatorname{Perm}(n): A^{F_{P}(\cdot)}=1\right)$. Then

$$
P_{F}=\sum_{Y \in \mathbf{v}_{\text {one }}} p_{F} \geq \sum_{Y \in\left(\mathbf{v}_{\text {one }} \cap \mathbf{v}_{\text {dist }}\right)} p_{F} \geq\left(1-\frac{q^{3}(w+1)^{4}}{2^{2 n+1}}\right) \sum_{Y \in\left(\mathbf{Y}_{\text {one }} \cap \mathbf{Y}_{\text {dist }}\right)} \frac{1}{\left(2^{n w}\right)^{q}}
$$

where the last inequality follows from Lemma 1. Then $P_{F}$ is at least

$$
\left(1-\frac{q^{3}(w+1)^{4}}{2^{2 n+1}}\right) \frac{\left|\mathbf{v}_{\text {one }} \cap \mathbf{v}_{\text {dist }}\right|}{\left(2^{n w}\right)^{q}} \geq\left(1-\frac{q^{3}(w+1)^{4}}{2^{2 n+1}}\right)\left(P_{R}-\frac{q w(w+1)}{2^{n+1}}\right)
$$

from (6) and (7). Now, we have $P_{F} \geq P_{R}-q^{3}(w+1)^{4} / 2^{2 n+1}-q w(w+1) / 2^{n+1}$, and by applying the same argument to $1-P_{F}$ and $1-P_{R}$, we have $1-P_{F} \geq$ $1-P_{R}-q^{3}(w+1)^{4} / 2^{2 n+1}-q w(w+1) / 2^{n+1}$.

\section{B Proof of Theorem 2}

Proof (of Theorem 2). Suppose for a contradiction that $\mathbf{A d v}_{\mathrm{CENC}}^{\mathrm{priv}}(A)$ is larger than the right hand side of (3) . Let the oracle $\mathcal{O}$ be either $F_{P}^{+}(\cdot)$ or $R(\cdot) \in$ Func $(n, n w)$. Consider the PRF-adversary $B$ for $F^{+}$in Figure 6 , where $B$ uses the nonce-respecting PRIV-adversary $A$ for CENC as a subroutine.

We see that if $\mathcal{O}$ is $F_{P}^{+}(\cdot)$, then $B$ gives $A$ a perfect simulation of CENC.Enc, since $F_{P}^{+}(\cdot)$ corresponds to "one frame" of CENC.KSGen, and therefore the outputs of CENC.KSGen.Sim(ctr,$l)$ and CENC.KSGen $P(\operatorname{ctr}, l)$ are the same. This implies $\operatorname{Pr}\left(P \stackrel{R}{\leftarrow} \operatorname{Perm}(n): B^{F_{P}^{+}(\cdot)}=1\right)=\operatorname{Pr}\left(P \stackrel{R}{\leftarrow} \operatorname{Perm}(n): A^{\operatorname{CENC}^{-E_{n}}(\cdot, \cdot)}=\right.$ $1)$. Also, it is easy to check that $B$ is input-respecting. On the other hand, if $\mathcal{O}$ is $R(\cdot)$, then $B$ gives $A$ a perfect simulation of $\mathcal{R}$. That is, $\operatorname{Pr}(R \stackrel{R}{\leftarrow} \operatorname{Func}(n, n w)$ : $\left.B^{R(\cdot)}=1\right)=\operatorname{Pr}\left(A^{\mathcal{R}(\cdot, \cdot)}=1\right)$. Therefore, we have $\mathbf{A d v}_{F^{+}}^{\text {prf }}(B)=\mathbf{A d v}_{\text {CENC }}^{\text {priv }}(A)$.

Suppose that the queries made by $A$ are $\left(N_{0}, M_{0}\right), \ldots,\left(N_{q-1}, M_{q-1}\right)$. If we let $l_{i}=\left\lceil\left|M_{i}\right| / n\right\rceil$, then $B$ makes $\left\lceil l_{0} / w\right\rceil+\cdots+\left\lceil l_{q-1} / w\right\rceil$ queries, which is at most $\left(l_{0}+\cdots+l_{q-1}\right) / w+q \leq \sigma / w+q=\hat{\sigma} / w$ queries. Note that this holds regardless of the value of $l_{0}, \ldots, l_{q-1}$. From the assumption for a contradiction, $\mathbf{A d v}_{\mathrm{CENC}}^{\text {priv }}(A)$ is larger than the right hand side of (3), which implies $\mathbf{A d v}_{F^{+}}^{\text {prf }}(B)>(w+$ $1)^{4} \hat{\sigma}^{3} / w^{3} 2^{2 n+1}+(w+1) \hat{\sigma} / 2^{n+1}$. This contradicts Corollary 1. 